\title{
Estimation of Gene Effects Based on Joint Scaling Test and Sequential Model Fit Scheme for Quantitative Traits in Sesame (Sesamum indicum L.)
}

\author{
Jawahar Lal Jatothu ${ }^{1}$, Kuldeep Singh Dangi ${ }^{2}$, Sudheer Kumar Sagi ${ }^{2}$, H. R. Bhandari ${ }^{3}$, M. K. Tripathi ${ }^{4}$ \\ $\&$ Babita Chaudhary ${ }^{4}$ \\ ${ }^{1}$ Scientist, Directorate of Oilseeds Research, Rajendranagar, Hyderabad, India \\ ${ }^{2}$ Professor, Department of Genetics and Plant Breeding, College of Agriculture, ANGRAU, Rajendranagar, \\ Hyderabad, India \\ ${ }^{3}$ Scientist, Central seed Research Station for Jute \& Allied Fibres, Bud Bud, Burdwan PIN, India \\ ${ }^{4}$ Senior Scientist, Sunnhemp Research Station, Pratapgarh, U.P. PIN, India \\ Correspondence: Jawahar Lal Jatothu, Directorate of Oilseeds Research, Rajendranagar, Hyderabad, India. Tel: \\ 91-916-045-1473. E-mail: jawaharlaljatoth@gmail.com
}

\author{
Received: August 1, 2012 Accepted: September 5, 2012 Online Published: February 17, 2013 \\ doi:10.5539/jas.v5n3p224 URL: http://dx.doi.org/10.5539/jas.v5n3p224
}

\begin{abstract}
Estimates of gene effects through joint scaling test of three and six parameter and sequential fit model in five crosses for eleven characters were investigated. It was noticed that simple additive dominance model exhibited lack of good fit for all the traits. So, sequential fit model was searched after eliminating the non-significant parameters of six parameter model. Five parameter sequential fit model was observed for number of primaries per plant (cross 1), number of seeds per capsule (cross 4), oil content (cross 2 and 3), seed yield per plant (cross 1) and chlorophyll content (cross 3). Best fit four parameter sequential model was observed for number of primaries per plant (cross 2) and 1000 seed weight (cross 1). Higher order interactions epistasis or linkage were observed for days to 50 per cent flowering (cross 1,2,3,4, and 5), days to maturity (cross 1, 2, 3, and 4), plant height (cross 3 , 4 , and 5), number of effective primaries per plant (cross 3,4 , and 5), number of effective capsules per plant (cross 2, 3, 4, and 5), number of seeds per capsule (cross 1, 2, 3, and 5), 1000 seed weight (cross 4 and 5), seed yield per plant (cross 4 and 5), oil content (cross 1, 4, and 5) and chlorophyll content (cross 1, 2, 4, and 5). Differential model schemes for same trait in different crosses were noticed in the present investigation. It was due to different parents involved with variable gene frequency with opposing and reinforcing genetic effects. The magnitude of [d] was relatively small to that of other genetic effects. This indicated that, additive genes are playing a minor role in the inheritance of these traits.
\end{abstract}

Keywords: sesame (Sesamum indicum L.), cross, capsule, oil content, seed yield, gene action, generation mean analysis

\section{Introduction}

Sesame (Sesamum indicum L.), commonly known as gingelly, til, simsim, a member of family pedaliaceae. It is probably the most ancient oilseed known and used by man and its domestication is lost in the mists of antiquity (Weiss 1983). Although originated in Africa, it spread early through West Asia to India, China and Japan which themselves became secondary distribution centers (Weiss, 1983). It is called as the "Queen of oil seeds" because of its excellent qualities of the seed, oil and meal. Sesame is highly nutritive (oil $50 \%$, protein $25 \%$ ) and its oil contains an antioxidant called sesamol which imparts a high degree of resistance against oxidative rancidity. Sesame cake is nutritious feed for dairy cattle and it can also be used as fertilizer (Ashri, 1989).

Sesame seeds are digestive, rejuvenative, antiaging, and rich in vitamins E, A and B complex and minerals like calcium, phosphorus, iron, copper, magnesium, zinc, and potassium. This unique composition coupled with high-unsaturated fatty acid (linolinic and tochopherol) make the sesame nearly perfect food (Lokesha \& Theertha, 2006). Sesame is the most neglected oil seed crop grown on marginal lands under poor management resulting in very low yield (Cagrgan, 2006). Further, the poor yield is due to the non-availability of cultivars to suit 
the diverse agro-climatic conditions. Hence, development of improved high yielding cultivars adapted to local conditions has become top priority.

It is an important annual oilseed crop in the tropics and warm subtropics, where it is usually grown in small patches (Bedigian \& Harlan, 1986). In India, sesame is cultivated in an area of 1.94 million ha with a production of 0.64 million tones annually (Damodaram \& Hegde, 2010). Being the fourth important oil seed crop in Indian agriculture after groundnut, rape seed and mustard, it is widely cultivated in the states of Uttar Pradesh, Rajasthan, Orissa, Gujarat, Andhra Pradesh, Tamil Nadu, Karnataka, West Bengal, Bihar and Assam.

India ranks second in area (1.94 million ha) and production (0.64 million tones) among the sesame growing countries. However, the productivity is low in India (332 kg/ha) as compared to world's average $(443 \mathrm{~kg} / \mathrm{ha})$ and it is far below as compared to Egypt $(1175 \mathrm{~kg} / \mathrm{ha})$ being the highest. This evidence indicates the potentiality of the crop for improvement in yields. The present sesame varieties under cultivation have limited yield potential. Most of the varieties evolved and released for cultivation are selections from locals or closely related populations under low levels of management. This is the major cause attributed for low productivity potential of cultivars grown in India. This indicates the need to enhance the productivity of this crop by developing high yielding genotypes, which depend on the availability of variability for yield and its component traits in the populations. Sesame has a wide range of genetic variability in its extensive germplasm collections. However, certain highly desirable traits have not been found so far, including good seed retention and resistance to certain diseases (Ashri, 1998).

For genetic improvement of the crop, the breeding method to be adopted depends mainly on the nature of gene action involved in the expression of quantitative trait. Line $\mathrm{x}$ Tester $(\mathrm{L} \times \mathrm{T})$ analysis is used to select the parents based on their combining ability but fails to detect the epistasis, which remains the most complex problem and on which it is extremely difficult to obtain reliable results. The inherent draw back of $\mathrm{L} x \mathrm{~T}$ design is that, it estimates additive and dominance components of gene action only and information on epistasis cannot be estimated which is an integral component of genetic architecture of population. So, information on the presence of type of epistatic genetic efects in the inheritance of various quantitative traits is important for adopting suitable breeding procedures to improve the traits. Generation mean analysis (Hayman, 1958) gives a comprehensive picture of gene action controlling the trait. It is relatively a simple first degree statistically analyzed technique to know the predominant genetic efects that are responsible in effecting the variation of character. In this area of generation mean analysis in sesame very little work has been done in India and abroad.

Very little work has been done in this area of joint scaling test and sequential best fit model of generation mean analysis in sesame in India and abroad. Therefore, the present investigation was made with objective to estimate gene action through joint scaling test and sequential best fit model of generation mean analysis.

\section{Material and Methods}

Sixteen Sesamum indicum L. cultivars (IS 1547 A, KKS-98049, PKDS-62, SI-7818, JCS-720, JCS-724, KMR-108, KMR-24, S-0018, CST 2001-5, KMS 5-396, JCS-507, IS 562 B, SI-3171, KMR-78 and TKG-22) collected from different agro morphological regions, India were used as experimental material. These selected parents were crossed in L x T fashion to generate 60 hybrids in Kharif, 2007. These 60 crosses along with their parents and checks were evaluated in Rabi, 2007-08. From the above 60 crosses five promising crosses were selected and back crosses were made and also $\mathrm{F}_{1} \mathrm{~s}$ are selfed to produce $\mathrm{F}_{2}$ during kharif, 2008. All the six populations $\left(\mathrm{P}_{1}, \mathrm{P}_{2}, \mathrm{~F}_{1}, \mathrm{~F}_{2}\right.$, $\mathrm{BC}_{1}$ and $\mathrm{BC}_{2}$ ) were raised in randomized block design (RBD) with three replications in college farm, college of agriculture, Rajendranagar, Hyderabad during Rabi, 2008-09 and Kharif, 2009.

The experiments involved the six basic generations (the $P_{1}$ and $P_{2}$ parent cultivars, the $F_{1}$ and $F_{2}$ first and second filial generations, and the $\mathrm{BC}_{1}$ and $\mathrm{BC}_{2}$ first and second back crosses) of five combinations of the parental cultivars, these combinations being KMR-108 x JCS-507, KKS-98049 x IS 562 B, S-0018 x SI-3171, KKS-98049 x TKG-22 and CST 2001-5 x KMS 5-396. The segregating and non-segregating parental populations were cultivated in a Randomized block design with three replications at the Agricultural College farm, Rajendranagar, Hyderabad, India. The parents of the respective crosses were used as the male parent and the $F_{1}$ generation as the female parent and effected back crosses to produce the $B_{1}\left(F_{1}\right.$ back crossed to $\left.P_{1}\right)$ and $B_{2}\left(F_{1}\right.$ back crossed to $\left.P_{2}\right)$ generations and the $F_{1}$ hybrids were selfed to obtain $F_{2}$ seeds. All these generations were produced during two cropping seasons and, as such, all the six generations had to be grown together during the same cropping season. The row-length was always five meters but the number of rows varied as follows: three rows, for the non-segregating $\mathrm{P}_{1}, \mathrm{P}_{2}$ and $\mathrm{F}_{1} ; 40$ rows for the $\mathrm{F}_{2}$; and 20 rows for the $\mathrm{BC}_{1}$ and $\mathrm{BC}_{2}$ generations. Since the non-segregating generations represent the homogeneous population while the segregating generations represent the heterogeneous population. The sample size (i.e. number of plants analyzed) varied as follows: 30 plants for the 
$\mathrm{P}_{1}, \mathrm{P}_{2}$ and $\mathrm{F}_{1}$ generations, 400 plants for the $\mathrm{F}_{2}$ generations and 200 plants in the $\mathrm{BC}_{1}$ and $\mathrm{BC}_{2}$ generations. The traits assessed were days to $50 \%$ flowering, number of days to maturity, plant height $(\mathrm{cm})$, number of primaries per plant, number of capsules per plant, capsule length $(\mathrm{cm})$, number of seeds per capsule, weight of 1000 seeds $(\mathrm{g})$, seed yield per plant (g), oil content (\%) and chlorophyll content (SPAD units).

In addition to testing in A, B, C and D scales, data was further subjected to joint scaling test Cavalli (1952) in view of the advantages associated with this test. First it was fitted into 3 parameter model, when $\chi^{2}$ value was significant, it indicated that data does not fit into additive - dominance model, hence, sequential model was followed. The data was first subjected to 6 parameter model, and when all the components were significant in six parameter model, no further analysis was carried out. In the event of non significance of any one of the components, the same was deleted and the data fitted into 5 parameter model. This procedure was followed until the $\chi^{2}$ value is non significant and all the components were significant in the model. Fitness in 5 or 4 parameter model indicated the presence of digenic non allelic interaction.

\subsection{Chi-square Test}

$\chi^{2}$ test was applied for testing the deviation of an observed segregation for theoretical observation. Chi-square was calculated using the formula.

$$
\chi^{2}=\frac{\sum(O-E)^{2}}{E}
$$

Where,

$$
\begin{aligned}
& O=\text { Observed frequency } \\
& E=\text { Expected frequency } \\
& \Sigma=\text { Summation of the data }
\end{aligned}
$$

If the calculated values of $\chi^{2}$ is significant at 5 per cent level of significance, we can say that the fit is poor, one or more observed frequencies are not in accordance with the hypotheses assumed and vice versa. So, it is also known as goodness of fit. The degree of freedom (d. $\mathrm{f}$ ) in $\chi^{2}$ test is ( $\left.\mathrm{n}-1\right)$. Where $\mathrm{n}=$ number of classes.

\section{Results}

Mean data on various characters recorded on different generations viz., parents, $\mathrm{F}_{1}, \mathrm{~F}_{2}, \mathrm{BC}_{1}$ and $\mathrm{BC}_{2}$ for five cross combinations of sesame were subjected to joint scaling test. The weighted least square technique was employed under three parameter viz., $\mathrm{m}$ (mean), d (additive) and $\mathrm{h}$ (dominance) and six parameters viz., $\mathrm{m}, \mathrm{d}, \mathrm{h}, \mathrm{i}$ (additive $\mathrm{x}$ additive), $\mathrm{j}$ (additive $\mathrm{x}$ dominance) and $\mathrm{l}$ (dominance $\mathrm{x}$ dominance) models. First, the data were subjected to three parameter model to test the adequacy of additive-dominance model. Whenever calculated $\chi^{2}$ at 3 degrees of freedom was found to be significant, as it indicates the inadequacy of the additive-dominance model, then it was subjected to six parameter model, to know the non-significant parameters. Search was done for the sequential best fit model scheme after eliminating non-significant parameters, wherein $\chi^{2}$ values become non-significant and all the tested parameters used in the model were found to be significant. The joint scaling test found to be more efficient in detection of epistasis compared to individual scaling tests, Ketata et al. (1976) had also concluded superiority of joint scaling test over the simple scaling tests in wheat. The estimates of different genetic components of generation mean in terms of three, six and best fit model for eleven characters of five crosses in sesame are furnished in Table 1 to 5. The results are elaborated cross wise for 11 characters.

\subsection{Cross 1 (KMR $108 \times$ JCS 507)}

Under six parameters $\mathrm{m},(\mathrm{d}),(\mathrm{h}),(\mathrm{i}),(\mathrm{j})$, and (l) components were significant for all the traits except number of effective primaries per plant, 1000 seed weight and seed yield per plant. Three parameter model exhibited lack of good fit for the characters studied in case of KMR-108 X JCS-507. Therefore, the data was subjected to joint scaling test of six parameter model. It was deciphered that none of the characters studied fit in to this six parameter model. Further the data were analyzed through sequential fit model by eliminating the non significant parameters (Table 1). 
Table 1. Estimation of gene effects based on joint scaling test and sequential best fit model applied for various characters in sesame

Cross 1: KMR-108 X JCS-507

\begin{tabular}{|c|c|c|c|c|c|c|c|}
\hline $\begin{array}{l}50 \% \\
\text { ring }\end{array}$ & Days to matutrity & $\begin{array}{l}\text { No. Of effective } \\
\text { primaries/plant }\end{array}$ & $\begin{array}{c}\text { No. of } \\
\text { seeds/capsule }\end{array}$ & 1000 seed weight & Seed yield/plant & Oil content & $\begin{array}{c}\text { Chlorophyll } \\
\text { content (SPAD } \\
\text { units) }\end{array}$ \\
\hline \multicolumn{8}{|c|}{3 Parameter model } \\
\hline \pm 0.10 & $95.70 * * \pm 0.15$ & $7.06^{* *} \pm 0.10$ & $63.11^{* *} \pm 0.25$ & $2.46^{* *} \pm 0.01$ & $12.52 * * \pm 0.14$ & $43.73 * * \pm 0.03$ & $42.03^{* *} \pm 0.21$ \\
\hline \pm 0.08 & $1.99 * * \pm 0.09$ & $-0.81 * * \pm 0.07$ & $-5.75 * * \pm 0.19$ & $-0.02 * * \pm 0.005$ & $5.53^{* *} \pm 0.12$ & $1.49 * * \pm 0.03$ & $3.31 * * \pm 0.22$ \\
\hline $20 \mathrm{NS}$ & $-1.91 * * \pm 0.30$ & $2.64 * * \pm 0.20$ & $-5.76^{* *} \pm 0.48$ & $0.06^{* *} \pm 0.02$ & $-0.65 * \pm 0.28$ & $-2.84 * * \pm 0.07$ & $7.02 * * \pm 0.39$ \\
\hline $71 * *$ & $86.15^{* *}$ & $648.40 * *$ & $131.33 * *$ & $1084.09 * *$ & $403.41 * *$ & $1324.16^{* *}$ & $30.22 * *$ \\
\hline \multicolumn{8}{|c|}{6 Parameter model } \\
\hline \pm 0.31 & $95.83^{* *} \pm 0.34$ & $12.17 * * \pm 0.28$ & $59.50^{* *} \pm 0.72$ & $3.29 * * \pm 0.03$ & $11.72 * * \pm 0.38$ & $38.72 * * \pm 0.13$ & $41.66^{* *} \pm 0.81$ \\
\hline \pm 0.15 & $2.83^{* *} \pm 0.21$ & $0.00 \pm 0.15 \mathrm{NS}$ & $-3.67 * * \pm 0.43$ & $0.005 \pm 0.01 \mathrm{NS}$ & $5.03^{* *} \pm 0.24$ & $1.32 * * \pm 0.04$ & $1.32 * * \pm 0.34$ \\
\hline \pm 0.87 & $-3.83 * * \pm 0.98$ & $-8.80 * * \pm 0.76$ & $6.14 * * \pm 2.05$ & $-1.94 * * \quad \pm 0.07$ & $0.41 \pm 1.12 \mathrm{NS}$ & $10.42 * * \pm 0.34$ & $11.95^{* *} \pm 2.37$ \\
\hline \pm 0.27 & $0.67 * \pm 0.27$ & $-5.47 * * \pm 0.23$ & $3.56^{* *} \pm 0.58$ & $-0.87 * * \quad \pm 0.02$ & $2.40 * * \pm 0.30$ & $4.93^{* *} \pm 0.12$ & $-3.60^{* *} \pm 0.74$ \\
\hline \pm 0.38 & $-2.33^{* *} \pm 0.47$ & $-2.80^{* *} \pm 0.34$ & $-7.09 * * \pm 0.98$ & $0.02 \pm 0.02 \mathrm{NS}$ & $1.43 * * \pm 0.55$ & $2.03 * * \pm 0.11$ & $2.51 * * \pm 0.96$ \\
\hline \pm 0.61 & $3.00 * * \pm 0.76$ & $5.60 * * \pm 0.55$ & $-9.82 * * \pm 1.48$ & $1.22 * * \pm 0.07$ & $0.53 \pm 0.81 \mathrm{NS}$ & $-8.50 * * \pm 0.27$ & $-6.90 * * \pm 1.63$ \\
\hline \multicolumn{8}{|c|}{5 Parameter model } \\
\hline & - & $12.17^{* *} \pm 0.28$ & - & $3.28 * * \pm 0.03$ & $11.85^{* *} \pm 0.07$ & - & - \\
\hline & - & - & - & - & $5.02 * * \pm 0.24$ & - & - \\
\hline & - & $-8.80 * * \pm 0.73$ & - & $-1.94 * * \quad \pm 0.07$ & - & - & - \\
\hline & - & $-5.47 * * \pm 0.23$ & - & $-0.81 * * \quad \pm 0.02$ & $2.31 * * \pm 0.20$ & - & - \\
\hline & - & $-2.80 * * \pm 0.16$ & - & $0.03 \pm 0.01 \mathrm{NS}$ & $1.43 * * \pm 0.55$ & - & - \\
\hline & - & $5.60 * * \pm 0.53$ & - & $1.22 * * \pm 0.07$ & $0.82 * * \pm 0.23$ & - & - \\
\hline \multicolumn{8}{|c|}{4 Parameter model } \\
\hline & - & - & - & $3.28 * * \pm 0.03$ & - & - & - \\
\hline & - & - & - & $-1.91 * * \quad \pm 0.07$ & - & - & - \\
\hline & - & - & - & $-0.81 * * \quad \pm 0.02$ & - & - & - \\
\hline & - & - & - & $1.20 * * \pm 0.07$ & - & - & - \\
\hline & - & - & - & $3.68 \mathrm{NS}$ & - & - & - \\
\hline
\end{tabular}

** significant at $1 \%$ level, NS: Non significant, $\mathrm{m}$ : mean, $\mathrm{d}$ : additive, h:dominance, $\mathrm{i}$ : additive $\mathrm{x}$ additive, $\mathrm{j}$ : additive $\mathrm{x}$ dominance, 1 : dominance $\mathrm{x}$ dominance

\subsection{Cross 2 (KKS $98049 x$ IS 562 B)}

The characters were analyzed through six parameter model and sequential model as 3 parameter model exhibited lack of good fit. For the character oil content 5 parameter and for number of effective primaries per plant 4 parameter models were found to be best fit. Additive (d), dominance (h) and additive x dominance (j) type of gene interactions were playing significant role for the character oil content with predominance of $(\mathrm{h})$ and (l) components possessing opposite sign. The trait number of effective primaries per plant was mainly affected by dominance (h) type of gene action.

The remaining characters viz., days to $50 \%$ flowering, days to maturity, number of capsules per plant, number of seeds per capsule and chlorophyll content exhibited significance for all the tested six parameters suggesting the presence of linkage or higher order epistatic interactions (Table 2). 
Table 2. Estimation of gene effects based on joint scaling test and sequential best fit model applied for various characters in sesame

Cross 2. KKS-98049 X IS-562 B

\begin{tabular}{|c|c|c|c|c|c|c|}
\hline $\begin{array}{l}\text { Paramet } \\
\text { er }\end{array}$ & $\begin{array}{l}\text { Days to } 50 \% \\
\text { flowering }\end{array}$ & Days to matutrity & $\begin{array}{l}\text { No. Of effective } \\
\text { primaries/plant }\end{array}$ & Capsules/plant & $\begin{array}{c}\text { No.of } \\
\text { seeds/capsules }\end{array}$ & Oil content \\
\hline \multicolumn{7}{|c|}{3 Parameter model } \\
\hline $\mathrm{m}$ & $40.44 * * \pm 0.14$ & $96.67 * * \pm 0.08$ & $7.48 * * \pm 0.06$ & $94.98 * * \pm 0.77$ & $57.84 * * \pm 0.29$ & $39.76 * * \pm 0.05$ \\
\hline [d] & $0.63^{* *} \pm 0.10$ & $-3.34 * * \pm 0.07$ & $0.02 \pm 0.05 \mathrm{NS}$ & $2.38^{* *} \pm 0.43$ & $-2.42 * * \pm 0.31$ & $5.40 * * \pm 0.04$ \\
\hline$[\mathrm{h}]$ & $-3.06^{* *} \pm 0.29$ & $-3.66^{* * \pm 0} 0.17$ & $1.98 * * \pm 0.13$ & $23.13 * * \pm 1.58$ & $-13.75 * * \pm 0.55$ & $1.40 * * \pm 0.09$ \\
\hline$\chi^{2}(3 \mathrm{df})$ & $135.96^{* *}$ & $269.50 * *$ & $177.72 * *$ & $3061.55 * *$ & $345.93 * *$ & $773.78 * *$ \\
\hline \multicolumn{7}{|c|}{6 Parameter model } \\
\hline $\mathrm{m}$ & $43.17 * * \pm 0.44$ & $91.50 * * \pm 0.36$ & $8.20 * * \pm 0.21$ & $203.33 * * \pm 2.31$ & $39.70 * * \pm 1.56$ & $38.85^{* *} \pm 0.16$ \\
\hline [d] & $-0.50 * * \pm 0.15$ & $-3.50 * * \pm 0.09$ & $0.00 \pm 0.08 \mathrm{NS}$ & $-11.90 * * \pm 0.84$ & $-2.27 * * \pm 0.35$ & $5.32 * * \pm 0.06$ \\
\hline$[\mathrm{h}]$ & $-11.17 * * \pm 1.21$ & $7.50 * * \pm 0.91$ & $1.43 * \pm 0.61$ & $-218.00 * * \pm 5.92$ & $31.15^{* *} \pm 4.55$ & $6.28 * * \pm 0.46$ \\
\hline [i] & $-2.67 * * \pm 0.41$ & $5.33 * * \pm 0.35$ & $-1.20 * * \pm 0.19$ & $-111.40 * * \pm 2.16$ & $20.64 * * \pm 1.53$ & $-0.07 \pm 0.15 \mathrm{NS}$ \\
\hline$[\mathrm{j}]$ & $5.00^{* *} \pm 0.44$ & $1.67 * * \pm 0.29$ & $-0.27 \pm 0.24 \mathrm{NS}$ & $38.67 * * \pm 1.96$ & $-5.21 * * \pm 1.58$ & $0.50 * * \pm 0.18$ \\
\hline$[1]$ & $5.67 * * \pm 0.10$ & $-5.00 * * \pm 0.70$ & $-1.13 * \pm 0.44$ & $135.87^{* *} \pm 5.52$ & $-22.73^{* *} \pm 3.10$ & $-5.57 * * \pm 0.33$ \\
\hline \multicolumn{7}{|c|}{5 Parameter model } \\
\hline $\mathrm{m}$ & - & - & $8.20 * * \pm 0.21$ & - & - & $38.78 * * \pm 0.06$ \\
\hline [d] & - & - & - & - & - & $5.32 * * \pm 0.06$ \\
\hline$[\mathrm{h}]$ & - & - & $1.43 * \pm 0.60$ & - & - & $6.47 * * \pm 0.20$ \\
\hline [i] & - & - & $-1.20 * * \pm 0.19$ & - & - & $0.53 * * \pm 0.16$ \\
\hline [j] & - & - & $-0.27 \pm 0.17 \mathrm{NS}$ & - & - & $-5.68 * * \pm 0.20$ \\
\hline$[1]$ & - & - & $-1.13 * \pm 0.44$ & - & - & \\
\hline \multicolumn{7}{|c|}{4 Parameter model } \\
\hline $\mathrm{m}$ & - & - & $7.98 * * \pm 0.15$ & - & - & - \\
\hline$[\mathrm{h}]$ & - & - & $2.09 * * \pm 0.43$ & - & - & - \\
\hline [i] & - & - & $-0.98 * * \pm 0.13$ & - & - & - \\
\hline$[1]$ & - & - & $-1.57 * * \pm 0.34$ & - & - & - \\
\hline$\chi^{2}(2 \mathrm{df})$ & - & - & $2.49 \mathrm{NS}$ & - & - & - \\
\hline
\end{tabular}

** significant at $1 \%$ level, NS: Non significant, $\mathrm{m}$ : mean, $\mathrm{d}$ : additive, h:dominance, $\mathrm{i}$ : additive $\mathrm{x}$ additive, $\mathrm{j}$ : additive $\mathrm{x}$ dominance, 1 : dominance $\mathrm{x}$ dominance

\subsection{Cross 3 (S $0018 \times$ SI 3171)}

The joint scaling test indicated that the assumption of additive-dominance genetic model was not satisfied in any one of the characters studied, wherein all the tested parameters and $\chi^{2}$ values were significant (Table 3 ). Therefore, the data was subjected to joint scaling test of six parameter model and sequential fit models by eliminating the non-significant parameters.

The 5 parameter model was best fitted for the characters like oil content and chlorophyll content. For the characters viz., days to $50 \%$ flowering, days to maturity, number of capsules per plant, number of seeds per capsule all the six components tested is found to be significant, suggesting higher order interaction of epistasis and linkage involved in the inheritance of these characters. 
Table 3. Estimation of gene effects based on joint scaling test and sequential best fit model applied for various characters in sesame.

Cross 3. S-0018 X SI-3171

\begin{tabular}{|c|c|c|c|c|c|c|c|}
\hline $\begin{array}{l}\text { Paramet } \\
\text { er }\end{array}$ & $\begin{array}{l}\text { Days to } 50 \% \\
\text { flowering }\end{array}$ & $\begin{array}{l}\text { Days to } \\
\text { matutrity }\end{array}$ & Plant height & $\begin{array}{c}\text { No. Of } \\
\text { effective } \\
\text { primaries/plant }\end{array}$ & $\begin{array}{c}\text { No. of } \\
\text { capsule/plant }\end{array}$ & $\begin{array}{c}\text { No. of } \\
\text { seeds/capsule }\end{array}$ & Oil content \\
\hline \multicolumn{8}{|c|}{3 Parameter model } \\
\hline $\mathrm{m}$ & $38.91 * * \pm 0.10$ & $94.68 * * \pm 0.11$ & $131.45^{* *} \pm 0.49$ & $7.11 * * \pm 0.05$ & $109.54 * * \pm 0.78$ & $67.32 * * \pm 0.40$ & $43.64 * * \pm 0.04$ \\
\hline [d] & $1.66^{* *} \pm 0.08$ & $0.58^{* *} \pm 0.08$ & $2.20 * * \pm 0.47$ & $0.56 * * \pm 0.04$ & $-18.38^{* *} \pm 0.55$ & $12.61 * * \pm 0.24$ & $-5.27 * * \pm 0.03$ \\
\hline$[\mathrm{h}]$ & $-2.93 * * \pm 0.21$ & $1.74 * * \pm 0.21$ & $-13.10 * *_{ \pm} 0.93$ & $2.30 * * \pm 0.10$ & $30.31 * * \pm 1.56$ & $-10.28 * * \pm 0.79$ & $-0.86^{* *} \pm 0.08$ \\
\hline$\chi^{2}(3 \mathrm{df})$ & $118.84 * *$ & $1354.93 * *$ & $1782.60 * *$ & $193.95 * *$ & $259.66^{* *}$ & $689.53 * *$ & $170.43^{* *}$ \\
\hline \multicolumn{8}{|c|}{6 Parameter model } \\
\hline $\mathrm{m}$ & $35.83 * * \pm 0.37$ & $87.17 * * \pm 0.37$ & $71.72 * * \pm 1.80$ & $6.22 * * \pm 0.15$ & $89.93 * * \pm 2.16$ & $46.05^{* *} \pm 1.08$ & $43.80^{* *} \pm 0.11$ \\
\hline [d] & $1.17^{* *} \pm 0.13$ & $3.17 * * \pm 0.13$ & $7.54 * * \pm 0.64$ & $-0.22 * * \pm 0.07$ & $-15.03 * * \pm 1.16$ & $6.70^{* *} \pm 0.53$ & $-4.93 * * \pm 0.05$ \\
\hline$[\mathrm{h}]$ & $4.50^{* *} \pm 0.95$ & $18.83 * * \pm 0.96$ & $136.36 * * \pm 5.04$ & $4.62 * * \pm 0.40$ & $98.27 * * \pm 6.02$ & $35.43 * * \pm 2.82$ & $-0.30 \pm 0.31 \mathrm{NS}$ \\
\hline [i] & $3.33^{* *} \pm 0.35$ & $8.67 * * \pm 0.35$ & $65.77 * * \pm 1.68$ & $0.47^{* *} \pm 0.13$ & $16.47 * * \pm 1.83$ & $20.60^{* *} \pm 0.94$ & $-0.47 * * \pm 0.09$ \\
\hline [j] & $2.33^{* *} \pm 0.33$ & $-7.00 * * \pm \quad 0.33$ & $-11.97 * * \pm 1.90$ & $2.10 * * \pm 0.16$ & $-16.00^{* *} \pm 2.68$ & $15.58^{* *} \pm 1.18$ & $-1.00^{* *} \pm 0.13$ \\
\hline$[1]$ & $-4.33^{* *} \pm 0.72$ & $-10.33^{* *} \pm 0.76$ & $-83.96 * * \pm 3.51$ & $-1.43 * * \pm 0.29$ & $-61.33^{* *} \pm 4.62$ & $-20.93 * * \pm 2.16$ & $-1.53 * * \pm 0.27$ \\
\hline \multicolumn{8}{|c|}{5 Parameter model } \\
\hline $\mathrm{m}$ & - & - & - & - & - & - & $43.70^{* *} \pm 0.09$ \\
\hline [d] & - & - & - & - & - & - & $-4.92 * * \pm 0.05$ \\
\hline$[\mathrm{h}]$ & - & - & - & - & - & - & - \\
\hline [i] & - & - & - & - & - & - & $-0.39^{* *} \pm 0.06$ \\
\hline [j] & - & - & - & - & - & - & $-1.01 * * \pm 0.13$ \\
\hline$[1]$ & - & - & - & - & - & - & $-1.77 * * \pm 0.13$ \\
\hline $\begin{array}{l}\chi^{2}(1 \\
\text { d.f. })\end{array}$ & - & - & - & - & - & - & $0.94 \mathrm{NS}$ \\
\hline
\end{tabular}

** significant at $1 \%$ level, NS: Non significant, $\mathrm{m}$ : mean, $\mathrm{d}$ : additive, h:dominance, $\mathrm{i}$ : additive $\mathrm{x}$ additive, $\mathrm{j}$ : additive $\mathrm{x}$ dominance, 1 : dominance $\mathrm{x}$ dominance

\subsection{Cross 4 (KKS $98049 \times T K G-22)$}

In cross KKS-98049 x TKG-22, additive-dominance genetic model showed non-significant $\chi^{2}$ values for all the characters studied (Table 4). Lack of goodness of three parameter model indicates the role of epistatic interactions. Thus the data was subjected to six parameter model of joint scaling test and sequential fit schemes for all the traits. After eliminating the non-significant parameters from the analysis, significant ones were subjected to sequential best fit model of joint scaling test. The character number of seeds per capsule was best fitted in the 5 parameter model with significance of $\mathrm{m},(\mathrm{d})$, (h), (i), and (l) components. The parameter (h) and (l) components had opposite sign in case of number of seeds per capsule.

\subsection{Cross 5 (CST 2001-5 x KMS 5-396)}

In three parameter model $\mathrm{m}(\mathrm{d})$ and $(\mathrm{h})$ was found to exhibit the lack of good fit for the characters studied, showing the significance of $\chi^{2}$ value, which revealed the presence of non allelic interactions (Table 5). Under six parameter model $\mathrm{m},(\mathrm{d}),(\mathrm{h}),(\mathrm{i}),(\mathrm{j})$, and (l) components were significant for the traits viz., days to $50 \%$ flowering, days to maturity, plant height, number of effective primaries per plant, number of effective capsules per plant, 1000 seed weight, seed yield per plant, oil content and chlorophyll content showing the presence of higher order interactions in this cross for these characters and discontinued from other analysis. 
Table 4. Estimation of gene effects based on joint scaling test and sequential best fit model applied for various characters in sesame.

Cross 4. KKS-98049 X TKG-22

\begin{tabular}{|c|c|c|c|c|c|c|c|c|c|c|}
\hline $\begin{array}{c}\text { Paramet } \\
\text { er }\end{array}$ & $\begin{array}{c}\text { Days to } 50 \% \\
\text { flowering }\end{array}$ & $\begin{array}{l}\text { Days to } \\
\text { matutrity }\end{array}$ & Plant height & $\begin{array}{c}\text { No. Of } \\
\text { effective } \\
\text { primaries/plant }\end{array}$ & Capsules/plant & $\begin{array}{c}\text { No. of } \\
\text { seeds/capsule }\end{array}$ & 1000 seed weight & Seed yield/plant & Oil content & $\begin{array}{l}\text { Chlorophyll } \\
\text { content (SPAD } \\
\text { units) }\end{array}$ \\
\hline \multicolumn{11}{|c|}{3 Parameter model } \\
\hline $\mathrm{m}$ & $40.06 * * \pm 0.11$ & $94.77 * * \pm 0.08$ & $136.10^{* *} \pm 0.54$ & $6.95 * * \pm 0.06$ & $114.27 * * \pm 0.76$ & $64.86^{* *} \pm 0.35$ & $2.75^{* * *} \pm 0.01$ & $14.04 * * \pm 0.15$ & $38.31 * * \pm 0.04$ & $41.80 * * \pm 0.16$ \\
\hline [d] & $-1.04 * * \pm 0.07$ & $-0.50^{* *} \pm 0.06$ & $16.10^{* *} \pm 0.50$ & $0.54^{* *} \pm 0.03$ & $-14.10^{* * *} \pm 0.55$ & $-7.38^{* *} \pm 0.26$ & $-0.08^{* *} \pm 0.01$ & $0.94^{* *} \pm 0.13$ & $5.74^{* *} \pm 0.02$ & $2.38^{* * *} \pm 0.14$ \\
\hline [h] & $-1.59^{* *} \pm 0.22$ & $1.82^{* *} \pm 0.18$ & $-7.86^{* *} \pm 1.05$ & $0.80^{* *} \pm 0.11$ & $6.83^{* *} \pm 1.47$ & $-7.44 * * \pm 0.71$ & $-0.26^{* *} \pm 0.02$ & $-2.63^{* *} \pm 0.28$ & $0.93^{* *} \pm 0.08$ & $4.57^{* *} \pm 0.31$ \\
\hline$\chi^{2}(3 \mathrm{df})$ & $2741.65^{* *}$ & $1074.48^{* *}$ & $313.24 * *$ & $366.71^{* *}$ & $1167.43^{* *}$ & $1313.35^{* *}$ & $407.05^{* *}$ & $502.63^{* *}$ & $20696.17^{* *}$ & $96.42^{* *}$ \\
\hline \multicolumn{11}{|c|}{6 Parameter model } \\
\hline $\mathrm{m}$ & $17.67^{* *} \pm 0.45$ & $85.17 * * \pm 0.32$ & $103.84 * * \pm 1.97$ & $8.85^{* *} \pm 0.15$ & $54.23 * * \pm 2.19$ & $23.80^{* * *} \pm 1.24$ & $1.88^{* *} \pm 0.05$ & $5.91 * * \pm 0.42$ & $30.58 * * \pm 0.09$ & $46.10^{* * \pm} \pm 0.53$ \\
\hline [d] & $-0.33^{*} \pm 0.13$ & $-1.17^{* *} \pm 0.09$ & $12.88^{* *} \pm 0.68$ & $0.28^{* *} \pm 0.09$ & $-13.20^{* *} \pm 1.20$ & $-7.73^{* *} \pm 0.40$ & $-0.12 * * \pm 0.02$ & $-0.61 * \pm 0.23$ & $4.32 * * \pm 0.06$ & $1.98^{* *} \pm 0.24$ \\
\hline [h] & $40.67 * * \pm 1.07$ & $25.50 * * \pm 0.79$ & $88.06^{* *} \pm 5.68$ & $-3.19^{* *} \pm 0.40$ & $197.20^{* *} \pm 6.19$ & $84.50^{* *} \pm 3.13$ & $1.10^{* *} \pm 0.14$ & $21.72 * * \pm 1.22$ & $22.28 * * \pm 0.28$ & $-7.46^{* *} \pm 1.50$ \\
\hline [i] & $22.67 * * \pm 0.44$ & $9.33^{* *} \pm 0.31$ & $29.23^{* *} \pm 1.85$ & $-2.13^{* *} \pm 0.12$ & $39.00^{* *} \pm 1.83$ & $41.10^{* *} \pm 1.17$ & $0.92 * * \pm 0.05$ & $6.48^{* *} \pm 0.35$ & $9.20^{* *} \pm 0.07$ & $-4.42^{* *} \pm 0.47$ \\
\hline [j] & $-2.00^{* *} \pm 0.30$ & $2.33^{* *} \pm 0.24$ & $20.40^{* *} \pm 2.14$ & $1.17^{* *} \pm 0.19$ & $22.60^{* *} \pm 2.82$ & $1.91 \pm 1.06 \mathrm{NS}$ & $0.32^{* *} \pm 0.05$ & $3.49^{* *} \pm 0.56$ & $-1.83^{* *} \pm 0.14$ & $2.22^{* *} \pm 0.62$ \\
\hline [l] & $-26.67 * * \pm 0.88$ & $-15.67^{* *} \pm 0.62$ & $-67.09 * * \pm 4.09$ & $1.77 * * \pm 0.29$ & $-146.27 * * \pm 4.41$ & $-48.00 * * \pm 2.35$ & $-1.36 * * \pm 0.09$ & $-17.25^{* *} \pm 0.87$ & $-12.37 * * \pm 0.21$ & $7.92^{* *} \pm 1.07$ \\
\hline \multicolumn{11}{|c|}{5 Parameter model } \\
\hline $\mathrm{m}$ & - & - & - & - & - & $23.73^{* *} \pm 1.24$ & - & - & - & - \\
\hline [d] & - & - & - & - & - & $-7.17 * * \pm 0.26$ & - & - & - & - \\
\hline [h] & - & - & - & - & - & $84.73^{* *} \pm 3.13$ & - & - & - & - \\
\hline [i] & - & - & - & - & - & $41.93^{* *} \pm 1.17$ & - & - & - & - \\
\hline [j] & - & - & - & - & - & - & - & - & - & - \\
\hline [1] & - & - & - & - & - & $-48.15^{* * \pm 2.35}$ & - & - & - & - \\
\hline $\begin{array}{l}\chi^{2}(1 \\
\text { d.f. })\end{array}$ & - & - & - & - & - & $3.25 \mathrm{NS}$ & - & - & - & - \\
\hline
\end{tabular}

** significant at $1 \%$ level, NS: Non significant, $\mathrm{m}$ : mean, d: additive, h:dominance, $\mathrm{i}$ : additive $\mathrm{x}$ additive, $\mathrm{j}$ : additive $\mathrm{x}$ dominance, 1 : dominance $\mathrm{x}$ dominance

Table 5. Estimation of gene effects based on joint scaling test and sequential best fit model applied for various characters in sesame

Cross 5. CST 2001-5 X KMS 5-396

\begin{tabular}{|c|c|c|c|c|c|c|c|}
\hline Plant height & $\begin{array}{l}\text { No. Of effective } \\
\text { primaries/plant }\end{array}$ & Capsules/plant & $\begin{array}{c}\text { No. of } \\
\text { seeds/capsule }\end{array}$ & $\begin{array}{l}1000 \text { seed } \\
\text { weight }\end{array}$ & Seed yield/plant & Oil content & $\begin{array}{c}\text { Chlorophyll } \\
\text { content (SPAD } \\
\text { units) }\end{array}$ \\
\hline $132.54 * * \pm 0.60$ & $8.32 * * \pm 0.03$ & $104.01 * * \pm 0.60$ & $64.83^{* *} \pm 0.30$ & $2.75^{* *} \pm 0.02$ & $12.56^{* *} \pm 0.10$ & $45.38^{* *} \pm 0.03$ & $41.68^{* *} \pm 0.29$ \\
\hline $6.66^{* *} \pm 0.41$ & $0.36^{* *} \pm 0.03$ & $7.99 * * \pm 0.57$ & $3.64 * * \pm 0.22$ & $0.20 * * \pm 0.01$ & $1.12 * * \pm 0.08$ & $-0.83 * * \pm 0.03$ & $4.46^{* *} \pm 0.13$ \\
\hline$-2.25 \pm 1.21 \mathrm{NS}$ & $-1.89 * * \pm 0.07$ & $2.51 * \pm 1.18$ & $-5.07 * * \pm 0.58$ & $0.48 * * \pm 0.03$ & $4.05^{* *} \pm 0.21$ & $-4.27 * * \pm 0.07$ & $3.84 * * \pm 0.58$ \\
\hline $1566.12 * *$ & $283.34 * *$ & $825.00 * *$ & $355.74 * *$ & $684.34 * *$ & $1977.85^{* *}$ & $2081.11 * *$ & $124.14 * *$ \\
\hline $78.59 * * \pm 1.67$ & $6.70^{* *} \pm 0.14$ & $42.87 * * \pm 2.32$ & $62.42 * * \pm 0.92$ & $5.25^{* *} \pm 0.10$ & $30.39^{* *} \pm 0.47$ & $40.47^{* *} \pm 0.11$ & $38.00^{* *} \pm 0.80$ \\
\hline$-4.11 * * \pm 0.73$ & $-0.27 * * \pm 0.05$ & $-2.87 * * \pm 0.88$ & $-1.95 * * \pm 0.41$ & $0.09 * * \pm 0.02$ & $2.51 * * \pm 0.11$ & $-0.93 * * \pm 0.04$ & $1.78 * * \pm 0.34$ \\
\hline $125.10 * * \pm 4.52$ & $2.47 * * \pm 0.38$ & $177.63 * * \pm 6.43$ & $-6.09 * \pm 2.45$ & $-4.95 * * \pm 0.21$ & $-38.07 * * \pm 1.17$ & $8.93^{* *} \pm 0.33$ & $16.30 * * \pm 1.98$ \\
\hline $51.72 * * \pm 1.50$ & $1.07 * * \pm 0.13$ & $50.60 * * \pm 2.15$ & $6.82 * * \pm 0.82$ & $-2.39 * * \pm 0.09$ & $-18.14 * * \pm 0.46$ & $4.87^{* *} \pm 0.11$ & $2.70 * * \pm 0.72$ \\
\hline $29.14 * * \pm 1.79$ & $1.47 * * \pm 0.14$ & $43.40^{* *} \pm 2.43$ & $13.66^{* *} \pm 0.99$ & $0.20 * * \pm 0.05$ & $-7.38 * * \pm 0.35$ & $-0.60^{* *} \pm 0.11$ & $6.98^{* *} \pm 0.75$ \\
\hline$-68.25 * * \pm 3.60$ & $-2.93 * * \pm 0.26$ & $\begin{array}{c}-121.80 * * \pm \\
4.87\end{array}$ & $5.10 * * \pm 1.82$ & $3.19 * * \pm 0.16$ & $24.53^{* *} \pm 0.85$ & $-8.67^{* *} \pm 0.33$ & $-11.38 * * \pm 1.58$ \\
\hline
\end{tabular}

** significant at $1 \%$ level, NS: Non significant, $\mathrm{m}$ : mean, d: additive, h:dominance, $\mathrm{i}$ : additive $\mathrm{x}$ additive, $\mathrm{j}$ : additive $\mathrm{x}$ dominance, $\mathrm{l}$ : dominance $\mathrm{x}$ dominance

\section{Discussion}

A summary of findings of different genetic components of mean expressed in terms of genetic parameters assessed for eleven characters in five crosses of sesame has been presented in Table 6. 
Table 6. Summary of estimates of gene effects based on joint scaling test of three parameter and sequential best fit model for

Five crosses in sesame

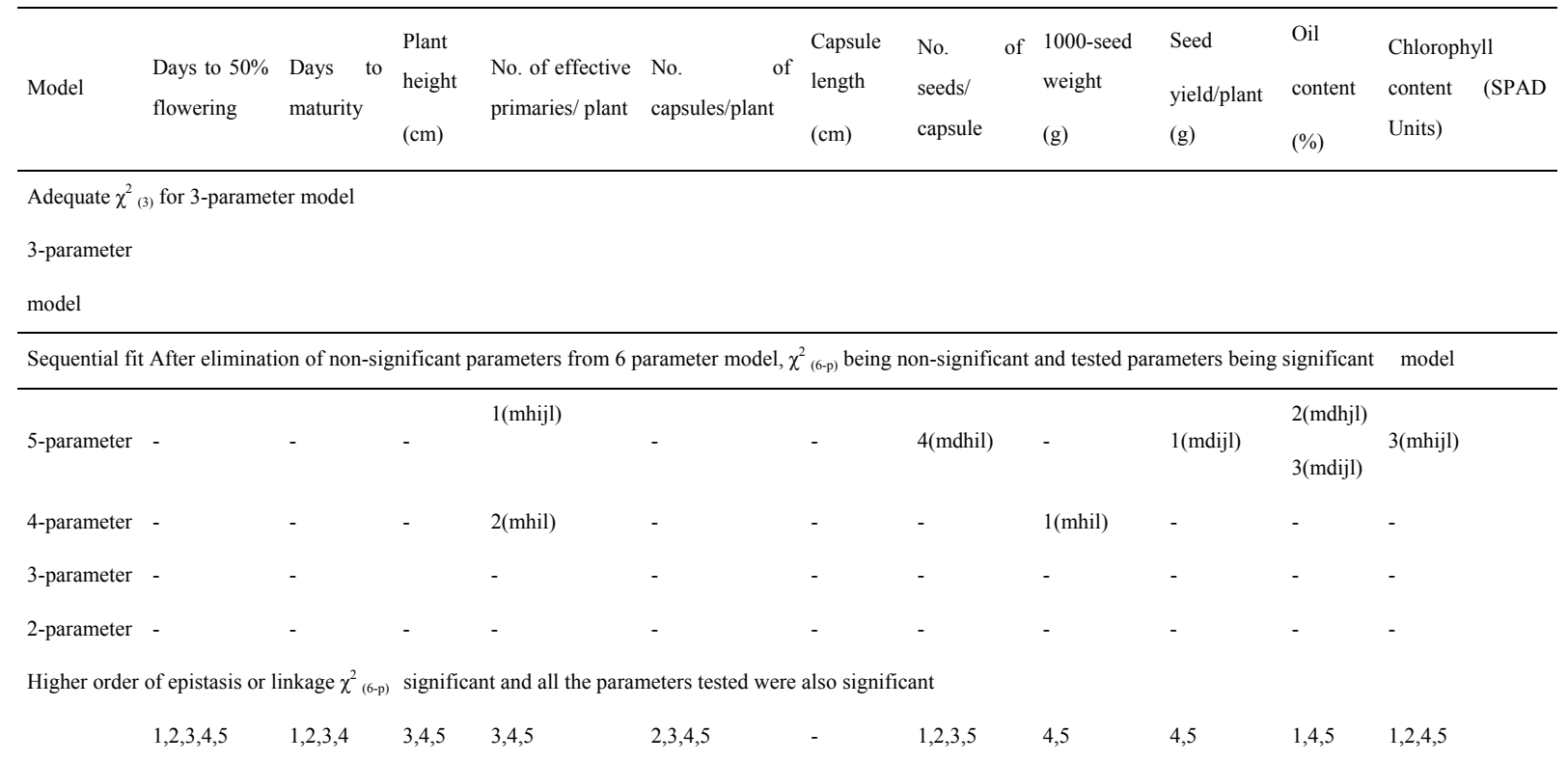

** significant at 1\% level, NS: Non significant, m: mean, d: additive, h:dominance, $\mathrm{i}$ : additive $\mathrm{x}$ additive, $\mathrm{j}$ : additive $\mathrm{x}$ dominance, 1 : dominance $\mathrm{x}$ dominance

1: Cross 1 (KMR-108 x JCS-507) 2: Cross 2 (KKS-98049 x TKG-22) 3: Cross 3 (KKS-98049 x IS 562 B) 4:

Cross 4 (CST 2001-5 x KMS 5-396 5: Cross 5 (S-0018 x SI-3171)

Search was made for the best sequential fit scheme and tested at alpha square (6-P) degrees of freedom. Five parameter sequential fit model was observed for number primaries per plant (cross 1), number seeds per capsule (cross 4), oil content (cross 2 and 3), seed yield per plant (cross 1) and chlorophyll content (cross 3). Best fit 4 parameter sequential model was observed for number primaries per plant (cross 2) and 1000 seed weight (cross 1).

Simple additive-dominance model exhibited lack of good fir for all the characters studied in all the crosses which indicated the presence of non-allelic interactions in all the traits. For number primaries per plant the joint scaling test of five parameter model exhibiting best fit in one cross(cross 1). The genetic parameters viz., m, (h), (i), (j), and (l) worked out are significantly important. In the above cross, dominance (h) and dominance x dominance (l) gene effects displayed opposite signs indicating duplicating type of epistasis where as cross 2 found to be the best fit for 4 parameter model. The genetic components $\mathrm{m}$, (h), (i), and (l) have played a significant role in revealing dominant (h) and dominant $\mathrm{x}$ dominant (l) type of gene actions as possessed opposite sign indicating the role of duplicate type of epistasis. Under sequential fit model the $\chi^{2}$ values in five crosses (cross 1,2,3,4, and 5) were found to be significant which indicates the presence of epistasis or linkage.

Regarding the number of seeds per capsule, non allelic interactions been observed in cross 4 . This cross exhibited the best fit for the sequential 5 parameter model. The genetic components $\mathrm{m},(\mathrm{d}),(\mathrm{h})$, (i), and (l) were significant in the above cross. In cross 4 duplicate type of epistasis was observed which was evident from possessing (h) and (l) components in the opposite signs. When sequential fit five parameter model was applied to cross 1, 2, 3, and 5, evidence of higher order epistasis or linkage can be predicted.

For the trait oil content sequential five parameter model was best fit for cross 2 and 3 . Under five parameter model, the components $\mathrm{m},(\mathrm{d}),(\mathrm{h}),(\mathrm{j})$, and (l) for cross 2 and $\mathrm{m},(\mathrm{d}),(\mathrm{i}),(\mathrm{j})$, and (l) for cross 3 were significant. Cross 2 exhibited relatively higher level of (h) and (l) components which has also possessed opposite signs indicating the prevalence of duplicate type of epistasis. Under five parameter model, higher order interactions could be observed in crosses 1,4 , and 5 .

For the character seed yield per plant, only in cross 1 sequential fit five parameter model was observed, where in genetic components dominance $(\mathrm{h})$, additive $\mathrm{x}$ additive (i), additive $\mathrm{x}$ dominance $(\mathrm{j})$ and dominance $\mathrm{x}$ dominance (l) were significant, where as in rest of the crosses (cross 4 and 5) all the tested parameters were significant and $\chi^{2}$ 
value is also significant. This suggests the involvement of higher order interactions or linkages or both in the inheritance of this trait.

In case of chlorophyll content, the cross 3 exhibited the best fit for sequential 5 parameter model where in genetic parameters $\mathrm{m},(\mathrm{h}),(\mathrm{i}),(\mathrm{j})$, and (l) were important. Dominance (h) and dominance x dominance (l) parameters possessed opposite sign indicating the role of duplicate gene action. For the crosses 1, 2, 4, and 5 evidence of higher order epistasis or linkage was predicted.

For 1000 seed weight, the cross 1 exhibited the best fit for the sequential 4 parameter model, the genetic components $\mathrm{m}$, (h), (i), and (l) found to be significantly important, where the genetic components (h) and (l) recorded relatively greater values with opposite signs indicating the prevalence of duplicate type of gene action. Two crosses viz., 4 and 5 exhibited significant $\chi^{2}$ values under sequential models which indicated the occurrence of higher order interactions epistasis or linkage.

In the present investigation occurrence of significance of common genetic parameters is a rare phenomenon observed in two or more crosses for the same trait. A considerable variation in the relative magnitude of different genetic parameters viz., $\mathrm{m},[\mathrm{d}],[\mathrm{h}],[\mathrm{i}],[\mathrm{j}]$, and [l] is observed from cross to cross for the same character, which may be attributed to the presence of variable frequency of genes with opposing and reinforcing effects in the parents involved in the crosses (Mather \& Jinks, 1982; Hallauer \& Miranda, 1989).

In the present study, magnitude of [d] is relatively small to that of other gene effects. This indicates that additive genes have lesser role in the inheritance of these traits. The material used in the present investigations is being derived from single crosses. As a result the material used gets selected in genetic background, additive gene effect is reduced (Gamble, 1962). In majority of the characters, the dominance gene action is playing a major role as compared to additive gene action. It is suggested that selection for high yielding genotypes be postponed till later generations to attain homozygous or selfing with reduction of dominance effect.

The importance of dominance gene effects is being indicated not only by its significance and relative magnitude but also by its sign. Positive dominance gene effects suggest its enhancing effects on the performance of different traits. However, for days to 50\% flowering crosses 1 and 2 dominance gene effects possessed negative sign, indicating that dominance is in direction of early maturity. In comparison to additive gene effect, dominant gene effect appeared to be more important for days to 50\% flowering (crosses 1, 3, 4, and 5), days to maturity (crosses 2 , 3 , and 4), plant height (crosses 3, 4, and 5), number of effective primaries per plant ( crosses 2, 3, and 5), number of capsules per plant ( cross 3, 4, and 5), number of seeds per capsule ( cross 1, 2, 3, 4, and 5), 1000 seed weight ( cross 4), oil content (cross 2, 4, and 5), chlorophyll content (cross 1, 2, 3, and 5) and seed yield per plant (cross 4).

The traits, days to $50 \%$ flowering (cross 1, 2, 3, 4, and 5), days to maturity (cross 1, 2, 3 and 4), plant height (cross 3,4 , and 5), number of effective primaries per plant cross 1, 2, 3, 4, and 5), number of capsules per plant (cross 2 , 3, 4, and 5), number of seeds per capsule ( cross 1, 2, 3, 4, and 5), 1000 seed weight ( cross 1, 4, and 5), oil content ( cross 1, 2, 4, and 5), chlorophyll content (cross 1, 2, 3, 4, and 5) and seed yield per plant (cross 4 and 5) were possessing opposite signs of (h) and (1) indicating the role of duplicate gene action controlling the traits (Table 7). Similar results have been obtained by Deenamani and Dorai Raj (1994) for number of capsules per plant and seed yield per plant, Ganesh and Sakila (1999) have recorded for plant height and seed yield per plant, Kumar, Thangavelu, and Rangaswamy (1998) for 1000 seed weight; Pathak and Dixit (1988) for days to maturity. Such type of duplicate type of gene action will pose hindrance to a plant breeder while attempting selection in long run.

In the complementary type of gene interactions particularly [i] and [1] reinforce the effect of dominance, while in the duplicate type of gene action, they oppose the effect of dominance component. It is for this reason, Jinks and Jones (1958) suggested that heterosis is likely to be expressed with greater magnitude in crosses where complementary type of interaction is observed, while it may not be observed at all in crosses showing duplicate type of gene action. In the present investigation none of the characters in all the five crosses exhibited complementary type of epistasis which is also noticed by Rajendrakumar and Raveendran (1999) and Iqbal and Nadeem (2003).

Besides additive gene action, significant epistatic component of additive $\mathrm{x}$ additive (i) indicates the preponderance of additivity over non-additivity. In such cases, in a particular cross to improve the trait pedigree method will be rewarding. Such interactions have been noticed in certain crosses for various traits in the present study viz., days to $50 \%$ flowering, number of effective primaries per plant, number of seeds per capsule and chlorophyll content (crosses 1, 2, 3, 4, and 5), days to maturity (crosses 1, 2, 3, and 4), plant height (crosses 2, 4, and 5), number of capsules per plant ( crosses 2, 3, 4, and 5), 1000 seed weight and seed yield per plant ( crosses 1, 4 and 5) and oil content (crosses 1, 3, 4, and 5) and these interactions would enhance the isolation of superior recombination's from the segregating generations of the crosses. 
Table 7: Direction of dominance gene $[\mathrm{h}]$ and dominance $\mathrm{x}$ dominance (l) for various characters in 5 crosses of sesame

\begin{tabular}{|c|c|c|c|c|c|c|}
\hline \multirow{2}{*}{ character } & \multirow{2}{*}{$\begin{array}{l}\text { Gene } \\
\text { action }\end{array}$} & \multicolumn{5}{|c|}{ Cross 1 to 5} \\
\hline & & 1 & 2 & 3 & 4 & 5 \\
\hline \multirow{2}{*}{ Days to $50 \%$ flowering } & {$[\mathrm{h}]$} & - & - & + & + & + \\
\hline & [1] & + & + & - & - & - \\
\hline \multirow{2}{*}{ Days to maturity } & {$[\mathrm{h}]$} & - & + & + & + & 0 \\
\hline & [1] & + & - & - & - & 0 \\
\hline \multirow{2}{*}{ Plant height $(\mathrm{cm})$} & {$[\mathrm{h}]$} & 0 & 0 & + & + & + \\
\hline & {$[1]$} & 0 & 0 & - & - & - \\
\hline \multirow{2}{*}{ No. of effective primaries/ plant } & {$[\mathrm{h}]$} & - & + & + & - & + \\
\hline & {$[1]$} & + & - & - & + & - \\
\hline \multirow{2}{*}{ No. of capsules/ plant } & {$[\mathrm{h}]$} & 0 & - & + & + & + \\
\hline & {$[1]$} & 0 & + & - & - & - \\
\hline \multirow{2}{*}{ Capsule length $(\mathrm{cm})$} & {$[\mathrm{h}]$} & 0 & 0 & 0 & 0 & 0 \\
\hline & {$[1]$} & 0 & 0 & 0 & 0 & 0 \\
\hline \multirow{3}{*}{ No. of seeds/ capsule } & {$[\mathrm{h}]$} & + & + & + & + & - \\
\hline & {$[1]$} & - & - & - & - & + \\
\hline & {$[\mathrm{h}]$} & - & 0 & 0 & + & - \\
\hline \multirow[t]{2}{*}{ 1000-seed weight (g) } & [1] & + & 0 & 0 & - & + \\
\hline & {$[\mathrm{h}]$} & 0 & 0 & 0 & + & - \\
\hline \multirow[t]{2}{*}{ Seed yield/plant (g) } & [1] & + & 0 & 0 & - & + \\
\hline & {$[\mathrm{h}]$} & + & + & 0 & + & + \\
\hline Oil content $(\%)$ & [1] & - & - & - & - & - \\
\hline \multirow[t]{2}{*}{ Chlorophyll content (SPAD Units) } & {$[\mathrm{h}]$} & + & + & + & - & + \\
\hline & [1] & - & - & - & + & - \\
\hline
\end{tabular}

$+=$ Significant positive direction

- = Significant negative direction

$0=$ Non-significant effect

The epistatic component of additive $\mathrm{x}$ dominance (j) gene effects were found to be of considerable importance and observed in certain crosses for days to 50\% flowering, oil content and chlorophyll content (crosses 1, 2, 3, 4, and 5), days to maturity ( crosses 1,2,3, and 4), plant height (crosses 3, 4, and 5), number of effective primaries per plant( crosses 1,3,4, and 5), number of capsules per plant ( crosses 2, 3, 4, and 5), number of seeds per capsule (crosses 1, 2, 3, and 5), 1000 seed weight (crosses 4 and 5) and seed yield per plant (crosses 1, 4, and 5). These findings are in agreement with the earlier findings of Esmail (2007).

Sequential model schemes found to be the best fit for all the characters in all the crosses which have two important advantages. Firstly it increases the precision with which the remaining parameters are estimated after the elimination of non-significant components and secondly it provides more degrees of freedom for $\chi^{2}$ test (Mather \& Jinks, 1982). The results of generation mean analysis indicated that, epistasis is the integral part of genetic architecture of the material used in the investigation and breeder cannot ignore it. The magnitude of epistasis could be biased by the presence of linkage especially [i] and [1] (Kempthorne, 1957), however, the effect of epistasis is basic genetic mechanism perhaps cannot be considered as negligible. Genetic models assuring negligible epistasis may be somewhat biased. The genetic parameters estimated provide a test for different type of gene action and are useful in giving information for the improvement of these traits. However, these genetic effects cannot be 
interpreted based on relative genetic variances. Also estimates obtained from each cross may be unique in varying degrees and may not be applicable to its parental population (Sprague, 1966).

The presence of dominance and epistatic effects for different traits in all the crosses would slow down the progress of selection. Hence, suggested the use of intermating of selector followed by visual selection in early segregating generations, which would simultaneously exploit both types of gene effects. Further, this approach is likely to break some undesirable linkages resulting in the establishment of rare useful recombinations.

\section{Conclusion}

The perusal of generation mean analysis indicated that epistasis is the integral part of genetic architecture of the present material used in the investigation and breeder cannot ignore it. Hence, it can be stated that, to break the linkage between gene constellations reciprocal recurrent selection or diallel selective mating systems are the breeding strategies proposed for the improvement of yield and its attributes in sesame.

\section{References}

Ashri, A. (1989). Sesame breeding objectives and approaches. In Omran, A. (Ed.), Oil Crops: Sunflower, Linseed and Sesame. IDRC-MR205E, IDRC, Ottawa.

Ashri, A. (1998). Sesame breeding. Plant Breed Rev, 16, 179-228.

Bedigian, D., \& Harlon, J. R. (1986). Evidence for the cultivation of sesame in the ancient world. Economic Botany, 40, 137-154. http://dx.doi.org/10.1007/BF02859136

Cagrgan, M. I. (2006). Selection and morphological characterization of induced determinate mutants in sesame. Field Crops Research, 96, 19-24. http://dx.doi.org/10.1016/j.fcr.2005.06.010

Cavalli, L. L. (1952). An analysis of linkages of quantities inheritance. In E. C. R. Reive, \& C. H. Waddington (Eds.) (pp. 135-144). London: HMSO.

Damodaram, T., \& Hegde, D. M. (2010). Oil seeds situation: A statistical compendium. Directorate of Oilseeds Research, Hyderabad.

Deenamani, I. E., \& Stephen D. M. (1994). Genetics of quantitative characters associated with capsules in sesame (Sesamum indicum L.). Madras Agricultural Journal, 81, 241-243.

Esmail, R. M. (2007). Genetic analysis of yield and its contributing traits in two intra-specific cotton crosses. $J$ Appl Sci Res, 3(12), 2075-2080.

Gamble, E. E. (1962). Gene effects in corn I: Separation and relative importance of gene effects for yield. Canadian Journal of Plant Science, 15, 77-78.

Ganesh, S. K., \& Sakila, M. (1999). Generation mean analysis in sesame (Sesamum indicum L.) crosses. Sesame and safflower newsletter, 14, 8-14.

Hallauer, A. R., \& Mirinda, J. B. (1989). Quantitative genetics in maize breeding (pp.107-111). IOWA State University Press/Amer.

Hayman, B. I. (1958). The separation of epistasis from additive and dominance variation in generation means. Heredity, 12, 371-390. http://dx.doi.org/10.1038/hdy.1958.36

Iqbal, M. Z., \& Nadeem, M. A. (2003). Generation mean analysis for seed cotton yield and number of sympodial branches per plant in cotton (Gossypium hirsutum L.). Asian Journal of Plant Science, 2(4), 395-399. http://dx.doi.org/10.3923/ajps.2003.395.399

Jinks, J. L., \& Jones, R. M. (1958). Estimation of the components of heterosis. Genetics, 43, 223-234.

Kempthorne, O. (1957). An introduction to genetic statistics. New York: John Wiley and Sons.

Ketata, H., Smith, E. L., Edwards, L. H., \& Mc New, R. W. (1976). Detection of epistasis, additive and dominance variation in winter wheat (Triticum aestivum L. em Thell.). Crop Science, 16, 1-14. http://dx.doi.org/10.2135/cropsci1976.0011183X001600010001x

Kumar, S. T., Thangavelu, S., \& Sree, R. (1998). Genetic analysis of seed size characters in sesame. Sesame and safflower newsletter, 13, 26-32.

Lokesha, R., \& Theartha, P. D. (2006). Transgenic sesame for nutritional quality maintenance a dream. International conference on biotechnology approaches for elevating malnutrition and human health (p. 69), UAS, Bengaluru.

Mather, K., \& Jinks, J. L. (1982). Biometrical Genetics (3rd ed.). London: Chapman and Hall Ltd. 
Pathak, H. C., \& Dixit, S. K. (1988). Genetic analysis of single stemmed sesame (Sesamum indicum L.). Indian Journal of Genetics, 48(3), 325-330.

Rajendrakumar, P., \& Raveendran, T. S. (1999). Genetic analysis of yield and quality traits in upland cotton (Gossypium hirsutum L.) through generation mean analysis. Indian Journal of Agricultural Sciences, 69(7), 538-540.

Sprague, G. R. (1966). Quantitative genetics in plant improvement. Symposium on Plant Breeding. Ames, IOWA: IOWA State University Press.

Weiss, E. A. (1983). Oilseed Crops. New York: Longman. 